\title{
Treatment of Iron-deficiency Anemia in Patients with Subclinical Hypothyroidism
}

\section{BACKGROUND}

- Subclinical hypothyroidism is associated with hypercholesterolemia, infertility, iron-deficiency anemia, and poor obstetric outcome.

- Recent studies have shown an association between iron-deficiency anemia and subclinical hypothyroidism, and treatment with levothyroxine can improve the response to iron salt.

- No prior studies have studied a combination of levothyroxine and iron salt in patients with irondeficiency anemia and subclinical hypothyroidism as compared to each agent as monotherapy.

\section{OBJECTIVE}

- To determine whether treatment of subclinical hypothyroidism and iron-deficiency anemia with a combination of levothyroxine and iron salt would be superior to each treatment as monotherapy.

\section{METHODS}

- Design: Single-center, double-blinded, randomized, active-controlled, parallel experimental trial. The length of the trial was 3 months.

- Inclusion criteria: New diagnosis of subclinical hypothyroidism (defined as TSH between 4.5-10 and normal free T4 and T3) and iron-deficiency anemia ( $\mathrm{Hgb}<12 \mathrm{~g} / \mathrm{dL}$ and ferritin $<25 \mu \mathrm{g} / \mathrm{L}$ for women; $\mathrm{Hgb}<13.5 \mathrm{~g} / \mathrm{dL}$ and ferritin $<30 \mu \mathrm{g} / \mathrm{L}$ for men).

- Exclusion criteria: Females with a menstrual period lasting more than 5 days or more than usual amount of bleeding; patients with genitourinary or GI blood loss; hemolytic anemia; prior thyroid, cardiovascular, respiratory, renal, and liver disease; any chronic diseases, including diabetes, uncontrolled HTN, adrenal insufficiency, other endocrine disorders; or any cause for anemia other than iron deficiency and subclinical hypothyroidism.

- 60 patients enrolled, 20 in each of 3 groups:

- Ferrous sulfate $65 \mathrm{mg} /$ day + matching placebo

- Levothyroxine $50 \mathrm{mcg} / \mathrm{day}+$ matching placebo

○ Ferrous sulfate $65 \mathrm{mg} /$ day + levothyroxine $50 \mathrm{mcg} /$ day

- Primary outcome measure: Change from baseline in hemoglobin

- Secondary outcome measures: Change from baseline in serum ferritin and TSH

- Power was not calculated in the study.

- Per-protocol data-handling method employed.

\section{RESULTS}

- For the primary outcome measure, the change in hemoglobin from baseline in $\mathrm{g} / \mathrm{dL}$, the iron group increased $0.005 \pm 0.39$, levothyroxine $0.26 \pm 0.675$, and iron + levothyroxine increased $1.17 \pm 0.59, p<0.0001$ for iron and levothyroxine versus either treatment alone.

- For the secondary outcome measure, change in ferritin from baseline in $\mathrm{ng} / \mathrm{mL}$, iron group increased $0.722 \pm 5.32$, levothyroxine $0.61 \pm 3.77$, and iron + levothyroxine $10.78 \pm 5.19$, , $p<0.0001$ for iron and levothyroxine versus either treatment alone. 
- For change in TSH, iron decreased TSH $0.580 \pm 1.67$, levothyroxine $5.75 \pm 1.4$, and iron + levothyroxine $5.12 \pm 2.25$. Both groups containing levothyroxine significantly decreased TSH compared to iron alone $(p<0.0001)$, and there was no statistical difference between the two levothyroxine-containing groups.

- $\quad$ For the before-and-after treatment parameters within each group, the only significant changes were TSH decrease in levothyroxine, and Hgb and ferritin increase and TSH decrease with iron + levothyroxine, all with $p<0.001$.

- The authors suggest a regimen of levothyroxine + iron salt in patients with coexisting subclinical hypothyroidism and iron-deficiency anemia.

\section{STRENGTHS}

- Double-blind, randomized, active-controlled, parallel study design

- No apparent bias

\section{LIMITATIONS}

- Low dosages of drugs employed

- No adverse event reporting

- Short duration of study

- Unclear recruitment techniques

\section{CONCLUSIONS}

- Currently, enough evidence exists to recommend that patients with iron deficiency anemia who do not respond to iron therapy should have other causes investigated, including subclinical hypothyroidism. In patients with both iron-deficiency anemia and subclinical hypothyroidism, it is reasonable to treat patients with both iron supplementation and levothyroxine.

- Further studies are needed to clarify the relationship between iron-deficiency anemia and thyroid function. Studies should be larger, longer in duration, multicenter, and take comorbidities into account. It's important to also take adverse events into consideration. In addition, Iron doses should be higher and levothyroxine dose titration should also be incorporated.

Reference: Ravanbod M, Asadipooya K, Kalantarhormozi M, Nabipour I, Omrani GR. Treatment of irondeficiency anemia in patients with subclinical hypothyroidism. Am J Med. 2013 May;126(5):420-4.

Prepared by: Kelly Wickline, Doctor of Pharmacy Candidate 